Homology, Homotopy and Applications, vol.9(1), 2007, pp.439-450

\title{
DEFORMATION OF LEIBNIZ ALGEBRA MORPHISMS
}

\author{
ASHIS MANDAL \\ (communicated by Jean-Louis Loday)
}

\begin{abstract}
We study formal deformations of Leibniz algebra morphisms. The associated deformation cohomology that controls deformations is constructed using the cochain complex defining Leibniz cohomology.
\end{abstract}

\section{Introduction}

The aim of this paper is to study an algebraic deformation theory of Leibniz algebra morphisms. Leibniz algebras and dialgebras were introduced by J.-L. Loday in connection with cyclic homology and Hochschild homology of matrix algebras [10, 11]. Leibniz algebras have been introduced as a non-antisymmetric analogue of Lie algebras. Leibniz algebras, dialgebras, and many others (e.g. Zinbiel, dendriform, etc.) have been studied in [12]. Each of these is an algebra over a suitable operad. For instance, Leibniz algebras are precisely the algebras over the binary quadratic operad Leib. The original deformation theory of algebraic structures was introduced by Gerstenhaber in his monumental work $[\mathbf{2}, \mathbf{3}, \mathbf{4}, \mathbf{5}, \mathbf{6}]$. Gerstenhaber considered the case of associative algebras. An algebraic deformation theory of associative algebra morphisms was studied by Gerstenhaber and Schack in $[\mathbf{7 , ~ 8 , ~ 9 ] . ~ S o o n ~ a f t e r , ~}$ deformation theory of various other structures and associated morphisms began to be studied by many mathematicians. The Lie algebra case has been studied by Nijenhuis and Richardson in $[\mathbf{1 5}, \mathbf{1 6}]$. Deformations of dialgebras have been studied in [14]. Recently, deformations of dialgebra morphisms have been studied by Donald Yau in [18]. He also studied deformations of Zinbiel algebra morphisms in [17]. In [1], David Balavoine studied formal deformations of algebras over a quadratic operad. In his paper, he explained how to obtain deformation theory for all the classical cases, including associative, Lie, and others, each of which is an algebra over a suitable operad. He also explained the case of Leibniz algebras using his theory. In this paper, we consider the relative version, namely, formal deformations of Leibniz algebra morphisms.

The paper is organised as follows. In Section 2, we recall the definitions of Leibniz algebra and its cohomology. In Section 3, we introduce the deformation complex of a Leibniz algebra morphism. In Section 4, we study the notion of deformation of a Leibniz algebra morphism, define the notion of equivalence of deformations,

Received September 11, 2006, revised February 22, 2007; published on March 28, 2007. 2000 Mathematics Subject Classification: 13D03, 13D10, 14 D15.

Key words and phrases: Leibniz algebra morphism, formal deformation, cohomology.

Copyright (C) 2007, International Press. Permission to copy for private use granted. 
and study rigidity. Obstruction cochains that arise in extending deformations of finite order with given infinitesimals are studied in the final section. One of the main results in any deformation theory is to prove that obstruction cochains are cocycles. In our context, this consists of two parts; one arising from deformations of the Leibniz algebras in question and the other from deformation of the morphism between the Leibniz algebras. In [1], David Balavoine showed that the obstruction cochains arising from deformations of Leibniz algebras are cocycles. The other part is done by a direct computation parallel to arguments given in $[\mathbf{1 7}, \mathbf{1 8}]$.

\section{Leibniz algebra and its cohomology}

In this section, we recall the definition of a Leibniz algebra and describe its cohomology. Let $\mathbb{K}$ be a fixed field.

Definition 2.1. A Leibniz algebra is a $\mathbb{K}$-module $L$, equipped with a bracket operation, which is $\mathbb{K}$-bilinear and satisfies the Leibniz identity:

$$
[x,[y, z]]=[[x, y], z]-[[x, z], y] \text { for } x, y, z \in L .
$$

Any Lie algebra is automatically a Leibniz algebra, since in the presence of antisymmetry, the Jacobi identity reduces to the Leibniz identity. Here are some more examples:

Example 2.2. Let $(L, d)$ be a differential Lie algebra with the Lie bracket [,]. Then $L$ is a Leibniz algebra with the bracket operation $[x, y]_{d}:=[x, d y]$. The new bracket on $L$ is called the derived bracket.

Example 2.3. On $\bar{T}(V)=V \oplus V^{\otimes 2} \oplus \cdots \oplus V^{\otimes n} \oplus \cdots$, there is a unique bracket that makes it into a Leibniz algebra and satisfies

$$
x_{1} x_{2} \cdots x_{n}=\left[\cdots\left[\left[x_{1}, x_{2}\right], x_{3}\right], \ldots, x_{n}\right] .
$$

This is the free Leibniz algebra over a $\mathbb{K}$-module $V$.

Example 2.4. Let $A$ be any associative $\mathbb{K}$-algebra equipped with a $\mathbb{K}$-module map $D: A \longrightarrow A$ satisfying $D(x(D y))=D x D y=D((D x) y)$ for $x, y \in A$. Then $[x, y]:=$ $x(D y)-(D y) x$ is a Leibniz bracket on $A$. Some examples of $D$ satisfying the above identity are as follows:

1. $D$ is an algebra map satisfying $D^{2}=D$.

2. $A$ is a superalgebra (that is, any $x \in A$ can be written uniquely as $x=x_{+}+$ $\left.x_{-}\right)$and $D(x)=x_{+}$.

3. $D$ is a square-zero derivation, that is,

$$
D(x y)=(D x) y+x(D y) \text { and } D^{2} x=0 \text { for } x, y \in A .
$$

Example 2.5. Let $L$ be a vector space spanned by $\left\{e_{1}, e_{2}, e_{3}\right\}$ over $\mathbb{K}$. Define a bilinear map [, ]: $L \times L \longrightarrow L$ by $\left[e_{1}, e_{3}\right]=e_{2}$ and $\left[e_{3}, e_{3}\right]=e_{1}$, all other products of basis elements being 0 . Then $(L,[]$,$) is a Leibniz algebra of dimension 3$ over $\mathbb{K}$. 
Let $L$ be a Leibniz algebra and $M$ be a representation of $L$. By definition ([13]), $M$ is a $\mathbb{K}$-module equipped with two actions (left and right) of $L$,

$$
\begin{gathered}
{[-,-]: L \times M \longrightarrow M \text { and }[-,-]: M \times L \longrightarrow M \text { such that }} \\
{[x,[y, z]]=[[x, y], z]-[[x, z], y]}
\end{gathered}
$$

holds whenever one of the variables is from $M$ and the others from $L$. In particular, $L$ is a representation of itself with the obvious action given by the bracket in $L$.

Definition 2.6. Let $L$ be a Leibniz algebra and $M$ be a representation of $L$. Let $C L^{n}(L ; M):=\operatorname{Hom}_{\mathbb{K}}\left(L^{\otimes n}, M\right), n \geqslant 0$, and

$$
\delta^{n}: C L^{n}(L ; M) \longrightarrow C L^{n+1}(L ; M)
$$

be the $\mathbb{K}$-morphism given by

$$
\begin{aligned}
\delta^{n} f\left(x_{1}, \ldots, x_{n+1}\right) & :=\left[x_{1}, f\left(x_{2}, \ldots, x_{n+1}\right)\right]+\sum_{i=2}^{n+1}(-1)^{i}\left[f\left(x_{1}, \ldots, \hat{x_{i}}, \ldots, x_{n+1}\right), x_{i}\right] \\
& +\sum_{1 \leqslant i<j \leqslant n+1}(-1)^{j+1} f\left(x_{1}, \ldots, x_{i-1},\left[x_{i}, x_{j}\right], x_{i+1}, \ldots, \hat{x_{j}}, \ldots, x_{n+1}\right) .
\end{aligned}
$$

Then $\left(C L^{*}(L ; M), \delta\right)$ is a cochain complex, $[\mathbf{1 3}]$, whose cohomology is denoted by $H L^{*}(L ; M)$, called the cohomology of the Leibniz algebra $L$ with coefficients in the representation $M$. For $M=L$, with the obvious action as mentioned above, the cohomology is denoted by $H L^{*}(L ; L)$.

\section{Deformation complex of a Leibniz algebra morphism}

In the present section, we introduce the deformation complex of a Leibniz algebra morphism. We shall see in the subsequent sections that the second and the third cohomologies associated to the complex encode all the information about deformations. Let $L$ and $M$ be Leibniz algebras over a field $\mathbb{K}$. To make our exposition simpler, we use the same notation $[-,-]$ for the brackets of $L$ and $M$.

Definition 3.1. A $\mathbb{K}$-linear map $f: L \longrightarrow M$ is said to be a Leibniz algebra morphism if it preserves the brackets. In other words, $f([x, y])=[f(x), f(y)]$ for $x, y \in L$.

Let $f: L \longrightarrow M$ be a Leibniz algebra morphism. We regard $M$ as a representation of $L$ via $f$, where the actions of $L$ on $M$, again denoted by $[-,-]$, are $[-,-]: L \times$ $M \longrightarrow M,[l, m]:=[f(l), m]$ and $[-,-]: M \times L \longrightarrow M,[m, l]:=[m, f(l)]$ for $l \in L$ and $m \in M$.

Define a cochain complex $\left(C L^{*}(f ; f), d\right)$ as follows. Set $C L^{0}(f ; f):=0$. For $n \geqslant 1$, the module of $n$-cochains is

$$
C L^{n}(f ; f):=C L^{n}(L ; L) \times C L^{n}(M ; M) \times C L^{n-1}(L ; M) .
$$

The coboundary $d^{n}: C L^{n}(f ; f) \longrightarrow C L^{n+1}(f ; f)$ is defined by the formula

$$
d^{n}(u, v ; w):=\left(\delta^{n} u, \delta^{n} v ; f u-v f-\delta^{n-1} w\right)
$$

for $(u, v ; w) \in C L^{n}(f ; f)$. Here the $\delta^{n}$ on the right-hand side are the coboundaries of the complexes defining Leibniz cohomology groups, the map $v f: L^{\otimes n} \longrightarrow M$ is the 
linear map defined by $v f\left(x_{1}, \cdots, x_{n}\right)=v\left(f\left(x_{1}\right), \cdots, f\left(x_{n}\right)\right)$, and $f u$ is the composition of maps. Observe that for $(u, v ; w) \in C L^{n}(f ; f), \delta^{n+1} \delta^{n} u=0=\delta^{n+1} \delta^{n} v$ and

$$
f \delta^{n} u-\left(\delta^{n} v\right) f-\delta^{n}\left(f u-v f-\delta^{n-1} w\right)=f \delta^{n} u-\left(\delta^{n} v\right) f-\delta^{n} f u+\delta^{n} v f=0 .
$$

Thus $d^{n+1} d^{n}(u, v ; w)=0$ for $n \geqslant 0$. Hence we obtain

Proposition 3.2. $\left(C L^{*}(f ; f), d\right)$ is a cochain complex.

The cochain complex $\left(C L^{*}(f ; f), d\right)$ is called the deformation complex of $f$, and the corresponding cohomology modules are denoted by

$$
H L^{n}(f ; f):=H^{n}\left(\left(C L^{*}(f ; f), d\right)\right) .
$$

The proof of the following proposition, which relates $H L^{*}(f ; f)$ to $H L^{*}(L ; L)$, $H L^{*}(M ; M)$, and $H L^{*}(L ; M)$, is similar to that of Proposition 3.3 in [18].

Proposition 3.3. If $H L^{n}(L ; L)=0=H L^{n}(M ; M)$, and $H L^{n-1}(L ; M)=0$, then so is $H L^{n}(f ; f)$.

From now on we shall omit superscripts for coboundaries. It should be clear from the context which coboundary is being used.

\section{Deformation, equivalence, and rigidity}

In this section, we study formal 1-parameter families of deformations of a Leibniz algebra morphism, define equivalence of deformations, and obtain a condition for rigidity. All the basic notions of deformation theory of algebraic structures and the associated morphisms are originally due to Gerstenhaber $[\mathbf{2}, \mathbf{3}, \mathbf{5}, \mathbf{6}]$, and Gerstenhaber and Schack $[\mathbf{7}, \mathbf{8}, \mathbf{9}]$. Here we briefly describe the analogous concepts related to deformation of Leibniz algebra morphisms. From now on we shall assume that $\mathbb{K}$ is a field of characteristic zero, and $f: L \longrightarrow M$ is a Leibniz algebra morphism. Let $\mathbb{K}[[t]]$ be the ring of formal power series in $t$ with coefficients in $\mathbb{K}$.

Recall from [1] that a formal 1-parameter family of deformations of a Leibniz algebra $L$ is a Leibniz bracket $\mu_{t}$ on the $\mathbb{K}[[t]]$-module $L_{t}=L \otimes_{\mathbb{K}} \mathbb{K}[[t]]$, where $\mu_{t}=$ $\sum_{i \geqslant 0} \mu_{i} t^{i}, \mu_{i} \in C L^{2}(L ; L)$ with $\mu_{0}$ being the original Leibniz bracket on $L$.

Definition 4.1. A deformation $\left(\mu_{t}, \nu_{t} ; f_{t}\right)$ of $f$ is a Leibniz algebra morphism $f_{t}: L_{t} \longrightarrow M_{t}$ of the form $f_{t}=\sum_{i \geqslant 0} f_{i} t^{i}$ where each $f_{i}: L \longrightarrow M$ is a $\mathbb{K}$-linear map with $f_{0}=f$, and $L_{t}=L \otimes_{\mathbb{K}} \mathbb{K}[[t]], M_{t}=M \otimes_{\mathbb{K}} \mathbb{K}[[t]]$ are formal 1-parameter family of deformations of $L$ and $M$ given by brackets $\mu_{t}=\sum_{i \geqslant 0} \mu_{i} t^{i}$ and $\nu_{t}=\sum_{i \geqslant 0} \nu_{i} t^{i}$, respectively.

Thus a triple $\left(\mu_{t}, \nu_{t} ; f_{t}\right)$, as given above, is a deformation of $f$ provided the following equalities hold:

$$
\begin{array}{ll} 
& \mu_{t}\left(x, \mu_{t}(y, z)\right)=\mu_{t}\left(\mu_{t}(x, y), z\right)-\mu_{t}\left(\mu_{t}(x, z), y\right) \text { for } x, y, z \in L \\
& \nu_{t}\left(x, \nu_{t}(y, z)\right)=\nu_{t}\left(\nu_{t}(x, y), z\right)-\nu_{t}\left(\nu_{t}(x, z), y\right) \text { for } x, y, z \in M \\
\text { and } & f_{t}\left(\mu_{t}(x, y)\right)=\nu_{t}\left(f_{t}(x), f_{t}(y)\right) \text { for } x, y \in L
\end{array}
$$

Now, expanding both sides of each of the equations in (1) and collecting coefficients 
of $t^{n}$, we see that (1) is equivalent to the system of equations

$$
\begin{aligned}
\text { (i) } \sum_{i+j=n} \mu_{i}\left(x, \mu_{j}(y, z)\right) & =\sum_{i+j=n}\left\{\mu_{i}\left(\mu_{j}(x, y), z\right)-\mu_{i}\left(\mu_{j}(x, z), y\right)\right\} \text { for } x, y, z \in L ; \\
\text { (ii) } \sum_{i+j=n} \nu_{i}\left(x, \nu_{j}(y, z)\right) & =\sum_{i+j=n}\left\{\nu_{i}\left(\nu_{j}(x, y), z\right)-\nu_{i}\left(\nu_{j}(x, z), y\right)\right\} \text { for } x, y, z \in M ; \\
\text { (iii) } \sum_{i+j=n} f_{i}\left(\mu_{j}(x, y)\right) & =\sum_{i+j+k=n} \nu_{i}\left(f_{j}(x), f_{k}(y)\right) \text { for } x, y \in L .
\end{aligned}
$$

Remark 4.2. For $n=0$, conditions $(i)$ and (ii) are equivalent to the usual Leibniz identity of $\mu_{0}$ and $\nu_{0}$ respectively, and (iii) is equivalent to the fact that $f$ is a Leibniz algebra morphism. For $n=1,(i)$ and (ii) are equivalent to $\delta \mu_{1}=0=\delta \nu_{1}$, and (iii) is equivalent to $f \mu_{1}-\nu_{1} f-\delta f_{1}=0$. Thus for $n=1,(i)-(i i i)$ are equivalent to saying that $\left(\mu_{1}, \nu_{1} ; f_{1}\right) \in C L^{2}(f ; f)$ is a cocycle. In general, for $n \geqslant 2,\left(\mu_{n}, \nu_{n} ; f_{n}\right)$ is just a 2-cochain in $C L^{2}(f ; f)$.

Definition 4.3. The 2-cochain $\left(\mu_{1}, \nu_{1} ; f_{1}\right)$ is called the infinitesimal of the deformation $\left(\mu_{t}, \nu_{t} ; f_{t}\right)$. More generally, if $\left(\mu_{i}, \nu_{i} ; f_{i}\right)=0$ for $1 \leqslant i \leqslant(n-1)$, and $\left(\mu_{n}, \nu_{n} ; f_{n}\right)$ is a non-zero cochain in $C L^{2}(f ; f)$, then $\left(\mu_{n}, \nu_{n} ; f_{n}\right)$ is called the $n$-infinitesimal of the deformation $\left(\mu_{t}, \nu_{t} ; f_{t}\right)$.

Proposition 4.4. The infinitesimal $\left(\mu_{1}, \nu_{1} ; f_{1}\right)$ of the deformation $\left(\mu_{t}, \nu_{t} ; f_{t}\right)$ is a 2 cocycle in $C L^{2}(f ; f)$. More generally, the $n$-infinitesimal $\left(\mu_{n}, \nu_{n} ; f_{n}\right)$ is a 2 -cocycle.

Proof. By definition, $d\left(\mu_{1}, \nu_{1} ; f_{1}\right)=\left(\delta \mu_{1}, \delta \nu_{1} ; f \mu_{1}-\nu_{1} f-\delta f_{1}\right)$. The result now follows from the above remark.

Let $\left(\mu_{t}, \nu_{t} ; f_{t}\right)$ and $\left(\tilde{\mu}_{t}, \tilde{\nu}_{t} ; \tilde{f}_{t}\right)$ be two deformations of $f$. A formal isomorphism between the deformations $\mu_{t}$ and $\tilde{\mu}_{t}$ of a Leibniz algebra $L$ is a power series $\Phi_{t}=$ $\sum_{i \geqslant 0} \phi_{i} t^{i}$, where each $\phi_{i}: L \longrightarrow L$ is a $\mathbb{K}$-linear map with $\phi_{0}=i d_{L}$, the identity map on $L$, such that $\tilde{\mu}_{t}(x, y)=\Phi_{t} \circ \mu_{t}\left(\Phi_{t}^{-1}(x), \Phi_{t}^{-1}(y)\right)$ for $x, y \in L$.

Definition 4.5. A formal isomorphism $\left(\Phi_{t}, \Psi_{t}\right):\left(\mu_{t}, \nu_{t} ; f_{t}\right) \longrightarrow\left(\tilde{\mu}_{t}, \tilde{\nu}_{t} ; \tilde{f}_{t}\right)$ between two deformations of a Leibniz algebra morphism $f: L \longrightarrow M$ consists of a pair of formal isomorphisms $\Phi_{t}: \mu_{t} \longrightarrow \tilde{\mu}_{t}$ and $\Psi_{t}: \nu_{t} \longrightarrow \tilde{\nu}_{t}$ of deformations of the Leibniz algebras $L$ and $M$ respectively such that $\tilde{f}_{t}=\Psi_{t} \circ f_{t} \circ \Phi_{t}{ }^{-1}$.

Definition 4.6. Two deformations $\left(\mu_{t}, \nu_{t} ; f_{t}\right)$ and $\left(\tilde{\mu}_{t}, \tilde{\nu}_{t} ; \tilde{f}_{t}\right)$ are said to be equivalent if and only if there exists a formal isomorphism

$$
\left(\Phi_{t}, \Psi_{t}\right):\left(\mu_{t}, \nu_{t} ; f_{t}\right) \longrightarrow\left(\tilde{\mu}_{t}, \tilde{\nu}_{t} ; \tilde{f}_{t}\right) .
$$

Definition 4.7. Any deformation of $f: L \longrightarrow M$ that is equivalent to the deformation $\left(\mu_{0}, \nu_{0} ; f\right)$ is said to be a trivial deformation.

Theorem 4.8. The cohomology class of the infinitesimal of a deformation $\left(\mu_{t}, \nu_{t} ; f_{t}\right)$ of $f: L \longrightarrow M$ is determined by the equivalence class of $\left(\mu_{t}, \nu_{t} ; f_{t}\right)$. 
Proof. Let $\left(\Phi_{t}, \Psi_{t}\right):\left(\mu_{t}, \nu_{t} ; f_{t}\right) \longrightarrow\left(\tilde{\mu}_{t}, \tilde{\nu}_{t} ; \tilde{f}_{t}\right)$ be an equivalence. Then $\tilde{\mu}_{t}=\Phi_{t} \circ$ $\mu_{t} \circ \Phi_{t}{ }^{-1}, \tilde{\nu}_{t}=\Psi_{t} \circ \nu_{t} \circ \Psi_{t}^{-1}$, and $\tilde{f}_{t}=\Psi_{t} \circ f_{t} \circ \Phi_{t}{ }^{-1}$. Expanding the above identities and comparing coefficients of $t$, we have that $\mu_{1}-\tilde{\mu}_{1}=\delta \phi_{1}, \nu_{1}-\tilde{\nu}_{1}=\delta \psi_{1}$, and $f_{1}-\tilde{f}_{1}=f \phi_{1}-\psi_{1} f$. Then it follows that $d\left(\phi_{1}, \psi_{1} ; 0\right)=\left(\mu_{1}, \nu_{1} ; f_{1}\right)-\left(\tilde{\mu}_{1}, \tilde{\nu}_{1} ; \tilde{f}_{1}\right)$.

Definition 4.9. A Leibniz algebra morphism $f: L \longrightarrow M$ is said to be rigid if and only if every deformation of $f$ is trivial.

Theorem 4.10. A non-trivial deformation of a Leibniz algebra morphism is equivalent to a deformation whose $n$-infinitesimal is not a coboundary for some $n \geqslant 1$.

Proof. Let $\left(\mu_{t}, \nu_{t} ; f_{t}\right)$ be a deformation of $f$ with $n$-infinitesimal $\left(\mu_{n}, \nu_{n} ; f_{n}\right)$, for some $n \geqslant 1$. Assume that there exists a 1 -cochain $(\phi, \psi ; m) \in C L^{1}(f ; f)$ with

$$
d(\phi, \psi ; m)=\left(\mu_{n}, \nu_{n} ; f_{n}\right) .
$$

We may assume that $m=0$, as $d(\phi, \psi ; m)=d(\phi, \psi+\delta m, 0)$. This yields $\mu_{n}=\delta \phi$, $\nu_{n}=\delta \psi$, and $f_{n}=f \phi-\psi f$. Set $\Phi_{t}=i d_{L}+\phi t^{n}$ and $\Psi_{t}=i d_{M}+\psi t^{n}$. We now have a deformation $\left(\tilde{\mu}_{t}, \tilde{\nu}_{t} ; \tilde{f}_{t}\right)$, where $\tilde{\mu}_{t}=\Phi_{t} \circ \mu_{t} \circ \Phi_{t}^{-1}, \tilde{\nu}_{t}=\Psi_{t} \circ \nu_{t} \circ \Psi_{t}^{-1}$, and $\tilde{f}_{t}=$ $\Psi_{t} \circ f_{t} \circ \Phi_{t}^{-1}$. For $x, y \in L$, the first equality gives $\tilde{\mu}_{t}\left(\Phi_{t}(x), \Phi_{t}(y)\right)=\Phi_{t}\left(\mu_{t}(x, y)\right)$. Expanding both sides of this equality and equating coefficients of $t^{i}, i \leqslant n$, we get $\tilde{\mu}_{i}=0,1 \leqslant i \leqslant n-1$, and $\tilde{\mu}_{n}=\mu_{n}-\delta \phi=0$. Similarly, the other two equalities yield $\tilde{\nu}_{i}=0$ and $\tilde{f}_{i}=0$ for $i \leqslant n$. Thus the given deformation is equivalent to a deformation $\left(\tilde{\mu}_{t}, \tilde{\nu}_{t} ; \tilde{f}_{t}\right)$ for which $\left(\tilde{\mu}_{i}, \tilde{\nu}_{i} ; \tilde{f}_{i}\right)=0$ for $1 \leqslant i \leqslant n$. Hence we can repeat the argument to kill off any infinitesimal that is a coboundary. So the process must stop if the deformation is non-trivial.

Corollary 4.11. If $H L^{2}(f ; f)=0$, then $f: L \longrightarrow M$ is rigid.

Remark 4.12. In view of Proposition 3.3, if $H L^{2}(L ; L)=0=H L^{2}(M ; M)$, and $H L^{1}(L ; M)=0$, then the morphism $f: L \longrightarrow M$ is rigid.

\section{Obstruction Cocycles}

In this section, we shall study the problem of realising a 2-cocycle in $C L^{2}(f ; f)$ as the infinitesimal of a deformation of $f$. This will be done by detecting any obstructions to extending a given deformation modulo $t^{k}$ to a deformation modulo $t^{k+1}$, $k \geqslant 1$. Let $N$ be a positive integer.

Definition 5.1. A deformation of $f: L \longrightarrow M$ of order $N$ is a triple $\left(\mu_{t}, \nu_{t} ; f_{t}\right)$ such that $\mu_{t}=\sum_{i=0}^{N} \mu_{i} t^{i}$ and $\nu_{t}=\sum_{i=0}^{N} \nu_{i} t^{i}$ are deformations modulo $t^{N+1}$ of $L$ and $M$ respectively, that is, $\mu_{t}$ and $\nu_{t}$ satisfy $(i)$ and (ii) respectively for $0 \leqslant i \leqslant N$, and $f_{t}=\sum_{i=0}^{N} f_{i} t^{i}$ with $f_{0}=f$, each $f_{i}: L \longrightarrow M$ being $\mathbb{K}$-linear, satisfies (iii) for $0 \leqslant i \leqslant N$.

If there exists a 2-cochain $\left(\mu_{N+1}, \nu_{N+1} ; f_{N+1}\right) \in C L^{2}(f ; f)$, such that the triple $\left(\tilde{\mu}_{t}, \tilde{\nu}_{t} ; \tilde{f}_{t}\right)$ with $\tilde{\mu}_{t}=\mu_{t}+\mu_{N+1} t^{N+1}, \tilde{\nu}_{t}=\nu_{t}+\nu_{N+1} t^{N+1}$, and $\tilde{f}_{t}=f_{t}+f_{N+1} t^{N+1}$ is a deformation of $f$ of order $(N+1)$, then we say that $\left(\mu_{t}, \nu_{t} ; f_{t}\right)$ extends to a deformation of $f$ of order $(N+1)$. 
Homology, Homotopy and Applications, vol. 9(1), 2007

Definition 5.2. Let $\left(\mu_{t}, \nu_{t} ; f_{t}\right)$ be a deformation of $f$ of order $N$. Consider the cochains $\theta(L) \in C L^{3}(L ; L), \theta(M) \in C L^{3}(M ; M)$, and $\theta(f) \in C L^{2}(L ; M)$ where

$$
\theta(L)(x, y, z)=\sum_{\substack{i+j=N+1 \\ i, j>0}}\left\{\mu_{i}\left(\mu_{j}(x, y), z\right)-\mu_{i}\left(\mu_{j}(x, z), y\right)-\mu_{i}\left(x, \mu_{j}(y, z)\right)\right\}
$$

for $x, y, z \in L, \theta(M)$ has an expression similar to $\theta(L)$ with $\mu$ replaced by $\nu$, and

$$
\theta(f)(x, y)=\sum^{\prime} \nu_{i}\left(f_{j}(x), f_{k}(y)\right)-\sum_{\substack{i+j=N+1 \\ i, j>0}} f_{i}\left(\mu_{j}(x, y)\right) \text { for } x, y \in L .
$$

Here,

$$
\sum^{\prime}=\sum_{\substack{i+j=N+1 \\ i, j>0} k=0}+\sum_{\substack{i+k=N+1 \\ i, k>0} j=0}+\sum_{\substack{j+k=N+1 \\ j, k>0} ;=0}+\sum_{\substack{i+j+k=N+1 \\ i, j, k>0}} .
$$

The 3-cochain $\theta\left(\mu_{t}, \nu_{t} ; f_{t}\right)=(\theta(L), \theta(M) ; \theta(f)) \in C L^{3}(f ; f)$ is called the obstruction cochain for extending the deformation $\left(\mu_{t}, \nu_{t} ; f_{t}\right)$ of $f$ of order $N$ to a deformation of $f$ of order $N+1$.

Observe that $\theta(L)$ is the obstruction for extending the deformation $\mu_{t}$ modulo $t^{N+1}$ of $L$ to a deformation modulo $t^{N+2}$. A similar remark holds for $\theta(M)$. Our primary goal is to show that the obstruction cochain as defined above is a cocycle. This will follow from $[\mathbf{1}]$ and by a direct computation following $[\mathbf{1 7}, \mathbf{1 8}]$.

Let $S_{n}$ be the symmetric group of $n$ symbols. Recall that a permutation $\sigma \in$ $S_{p+q}$ is called a $(p, q)$-shuffle, if $\sigma(1)<\sigma(2)<\cdots<\sigma(p)$, and $\sigma(p+1)<\sigma(p+2)<$ $\cdots<\sigma(p+q)$. We denote the set of all $(p, q)$-shuffles in $S_{p+q}$ by $\operatorname{Sh}(p, q)$.

Let $L$ be a Leibniz algebra. For $\alpha \in C L^{p+1}(L ; L)$ and $\beta \in C L^{q+1}(L ; L)$, define $\alpha \circ \beta \in C L^{p+q+1}(L ; L)$ by

$$
\begin{aligned}
& \alpha \circ \beta\left(x_{1}, \ldots, x_{p+q+1}\right) \\
& =\sum_{k=1}^{p+1}(-1)^{q(k-1)}\left\{\sum _ { \sigma \in S h ( q , p - k + 1 ) } \operatorname { s g n } ( \sigma ) \alpha \left(x_{1}, \ldots, x_{k-1}, \beta\left(x_{k}, x_{\sigma(k+1)}, \ldots, x_{\sigma(k+q)}\right),\right.\right. \\
& \left.\left.x_{\sigma(k+q+1)}, \ldots, x_{\sigma(p+q+1)}\right)\right\} .
\end{aligned}
$$

Then the graded cochain module $C L^{*}(L ; L)=\bigoplus_{p} C L^{p}(L ; L)$ equipped with the bracket

$$
[\alpha, \beta]=\alpha \circ \beta+(-1)^{p q+1} \beta \circ \alpha \text { for } \alpha \in C L^{p+1}(L ; L) \text { and } \beta \in C L^{q+1}(L ; L)
$$

is a graded Lie algebra [1]. It is straightforward to check that the above bracket on $C L^{*}(L ; L)$ is related to the coboundary map as follows.

Lemma 5.3. For $\alpha \in C L^{p+1}(L ; L), \delta \alpha=-\left[\alpha, \mu_{0}\right]$.

Now observe that in terms of the bracket on $C L^{*}(L ; L)$, the obstruction cochain 
$\theta(L)$ can be written as

$$
\theta(L)=\frac{1}{2} \sum_{\substack{i+j=N+1 \\ i, j>0}}\left[\mu_{i}, \mu_{j}\right]
$$

In [1], David Balavoine proved that for any Leibniz algebra $L$, the obstruction cochain $\theta(L)$ for extending a deformation modulo $t^{N+1}$ to a deformation modulo $t^{N+2}$ is a cocycle. This is done by using the above expression of $\theta(L)$, Lemma 5.3, and the Jacobi identity in the graded Lie algebra $\left(C L^{*}(L ; L),[-,-]\right)$.

Theorem 5.4. The obstruction cochain $\theta\left(\mu_{t}, \nu_{t} ; f_{t}\right)$ of a deformation $\left(\mu_{t}, \nu_{t} ; f_{t}\right)$ of $f$ of order $N$ is a 3 -cocycle in $C L^{3}(f ; f)$.

Proof. In view of the above observations, it remains to prove that $\delta \theta(f)-f \theta(L)$ $+\theta(M) f=0$. This is done by a direct computation parallel to the proof of the corresponding results in $[\mathbf{1 7}, \mathbf{1 8}]$. Note that for $x, y, z \in L$,

$$
\begin{aligned}
(f \theta(L)-\theta(M) f)(x, y, z) & =\sum f \mu_{i}\left(\mu_{j}(x, y), z\right)-\sum f \mu_{i}\left(\mu_{j}(x, z), y\right) \\
& -\sum f \mu_{i}\left(x, \mu_{j}(y, z)\right)-\sum \nu_{i}\left(\nu_{j}(f(x), f(y)), f(z)\right) \\
& +\sum \nu_{i}\left(\nu_{j}(f(x), f(z)), f(y)\right) \\
& +\sum \nu_{i}\left(f(x), \nu_{j}(f(y), f(z))\right),
\end{aligned}
$$

where we are using the abbreviated symbol $\sum$ for $\sum_{\substack{i+j=N+1 \\ i, j>0}}$ in (2) and in the subsequent computations. By (2.6) and (5.2) we have,

$$
\begin{aligned}
\delta \theta(f)(x, y, z)= & \sum^{\prime} \nu_{0}\left(f(x), \nu_{i}\left(f_{j}(y), f_{k}(z)\right)\right)-\sum \nu_{0}\left(f(x), f_{i} \mu_{j}(y, z)\right) \\
& +\sum^{\prime} \nu_{0}\left(\nu_{i}\left(f_{j}(x), f_{k}(z)\right), f(y)\right)-\sum \nu_{0}\left(f_{i} \mu_{j}(x, z), f(y)\right) \\
& -\sum^{\prime} \nu_{0}\left(\nu_{i}\left(f_{j}(x), f_{k}(y)\right), f(z)\right)+\sum \nu_{0}\left(f_{i} \mu_{j}(x, y), f(z)\right) \\
& -\sum^{\prime} \nu_{i}\left(f_{j} \mu_{0}(x, y), f_{k}(z)\right)+\sum f_{i} \mu_{j}\left(\mu_{0}(x, y), z\right) \\
& +\sum^{\prime} \nu_{i}\left(f_{j} \mu_{0}(x, z), f_{k}(y)\right)-\sum f_{i} \mu_{j}\left(\mu_{0}(x, z), y\right) \\
& +\sum^{\prime} \nu_{i}\left(f_{j}(x), f_{k} \mu_{0}(y, z)\right)-\sum f_{i} \mu_{j}\left(x, \mu_{0}(y, z)\right) .
\end{aligned}
$$

The main idea involved is to rewrite some of the sums on the right-hand side of (3) using $(i)-(i i i)$ for $0 \leqslant n \leqslant N$, in order to cancel terms appearing in (2). First consider the 7 th sum. Using (iii) for each $j$, we have

$$
f_{j} \mu_{0}(x, y)=\sum_{\substack{\alpha+\beta+\gamma=j \\ \alpha, \beta, \gamma \geqslant 0}} \nu_{\alpha}\left(f_{\beta}(x), f_{\gamma}(y)\right)-\sum_{\substack{p+q=j \\ 1 \leqslant q \leqslant j}} f_{p} \mu_{q}(x, y) .
$$


Homology, Homotopy and Applications, vol. 9(1), 2007

Substituting this expression for $f_{j} \mu_{0}(x, y)$, the 7 th sum on the right-hand side of $(3)$ becomes

$$
-\sum_{\substack{\alpha+\beta+\gamma=j \\ \alpha, \beta, \gamma \geqslant 0}}^{\prime} \nu_{i}\left(\nu_{\alpha}\left(f_{\beta}(x), f_{\gamma}(y)\right), f_{k}(z)\right)+\sum_{\substack{p+q=j \\ 1 \leqslant q \leqslant j}}^{\prime} \nu_{i}\left(f_{p} \mu_{q}(x, y), f_{k}(z)\right) .
$$

Here as explained in [18], the first sum of (4) is given by

$$
\begin{aligned}
& \sum_{\substack{\alpha+\beta+\gamma=j \\
\alpha, \beta, \gamma \geqslant 0}}^{\prime}=\sum_{\substack{i+\alpha+\beta+\gamma=N+1 \\
i,(\alpha+\beta+\gamma)>0 \\
k=0 ; \alpha, \beta, \gamma \geqslant 0}}+\sum_{\substack{i+k=N+1 \\
i, k>0 \\
\alpha=\beta=\gamma=0}} \\
& +\sum_{\begin{array}{c}
\alpha+\beta+\gamma+k=N+1 \\
k,(\alpha+\beta+\gamma)>0 \\
i=0 ; \alpha, \beta, \gamma \geqslant 0
\end{array}}+\sum_{\begin{array}{c}
i+\alpha+\beta+\gamma+k=N+1 \\
i,(\alpha+\beta+\gamma), k>0 \\
\alpha, \beta, \gamma \geqslant 0
\end{array}}
\end{aligned}
$$

and the second sum is given by

$$
\sum_{\substack{p+q=j \\ 1 \leqslant q \leqslant j}}^{\prime}=\sum_{\substack{i+p+q=N+1 \\ i, q>0 ; p \geqslant 0 \\ k=0}}+\sum_{\substack{p+q+k=N+1 \\ q, k>0 ; p \geqslant 0 \\ i=0}} \sum_{\substack{i+p+q+k=N+1 \\ i, k, q>0 ; p \geqslant 0}}
$$

Thus the first sum of (4) splits into four sums, the first of which can be written as

$$
\begin{aligned}
& -\sum_{\substack{i+\alpha+\beta+\gamma=N+1 \\
i, \alpha+\beta+\gamma)>0 \\
k=0 ; \alpha, \beta, \gamma \geqslant 0}} \nu_{i}\left(\nu_{\alpha}\left(f_{\beta}(x), f_{\gamma}(y)\right), f_{k}(z)\right) \\
& =-\sum_{\substack{i+\alpha=N+1 \\
i, \alpha>0}} \nu_{i}\left(\nu_{\alpha}(f(x), f(y)), f(z)\right)-\sum_{\substack{i+\alpha+\beta+\gamma=N+1 \\
i, \beta+\gamma)>0 \\
\alpha, \beta, \gamma \geqslant 0}} \nu_{i}\left(\nu_{\alpha}\left(f_{\beta}(x), f_{\gamma}(y)\right), f(z)\right) .
\end{aligned}
$$

Note that the first sum on the right-hand side of (7) appears in (2) as one of the three summands of $-\theta(M) f(x, y, z)$. Analogously we rewrite the 9 th sum and the 11th sum on the right-hand side of (3) using ( $i i i)$, as described above. In the 8th sum on the right-hand side of $(3)$, we use $(i)$ to substitute $\mu_{j}\left(\mu_{0}(x, y), z\right)$, obtaining

$$
\begin{aligned}
& \sum_{\substack{i+j=N+1 \\
i, j>0}} f_{i} \mu_{j}\left(\mu_{0}(x, y), z\right)=\sum_{\substack{i+j=N+1 \\
i, j>0}} f_{i} \mu_{j}\left(x, \mu_{0}(y, z)\right) \\
& +\sum_{\substack{i+j+k=N+1 \\
i, k>0 ; j \geqslant 0}} f_{i} \mu_{j}\left(x, \mu_{k}(y, z)\right)+\sum_{\substack{i+j=N+1 \\
i, j>0}} f_{i} \mu_{j}\left(\mu_{0}(x, z), y\right) \\
& +\sum_{\substack{i+j+k=N+1 \\
i, k>0 ; j \geqslant 0}} f_{i} \mu_{j}\left(\mu_{k}(x, z), y\right)-\sum_{\substack{i+j+k=N+1 \\
i, k>0 ; j \geqslant 0}} f_{i} \mu_{j}\left(\mu_{k}(x, y), z\right) .
\end{aligned}
$$

Observe that the first and the third sums on the right-hand side of (8) cancel with the 12 th and the 10 th sums respectively on the right-hand side of $(3)$. Next, note 
Homology, Homotopy and Applications, vol. 9(1), 2007

that the 2 nd sum on the right-hand side of (8) can be written as

$$
\sum_{k=1}^{N} \sum_{\substack{i+j+k=N+1 \\ i, j \geqslant 0}} f_{i} \mu_{j}\left(x, \mu_{k}(y, z)\right)-f \sum_{\substack{j+k=N+1 \\ j, k>0}} \mu_{j}\left(x, \mu_{k}(y, z)\right)
$$

and the 2nd sum of (9) appears in $f \theta(L)(x, y, z)$. Also, by (iii), the first sum splits as

$$
\begin{aligned}
& \sum_{k=1}^{N} \sum_{\substack{\alpha+\beta+\gamma+k=N+1 \\
\alpha, \beta, \gamma \geqslant 0}} \nu_{\alpha}\left(f_{\beta}(x), f_{\gamma} \mu_{k}(y, z)\right) \\
& =\sum_{\substack{i+j=N+1 \\
i, j>0}} \nu_{0}\left(f(x), f_{i} \mu_{j}(y, z)\right)+\sum_{\substack{p+q=k \\
1 \leqslant q \leqslant k}}^{\prime} \nu_{i}\left(f_{j}(x), f_{p} \mu_{q}(y, z)\right) .
\end{aligned}
$$

The first sum in the last step appears as the 2nd term on the right-hand side of (3) with opposite sign, whereas the other sum can be seen to appear in the new expression of the 11th sum on the right-hand side of (3) with opposite sign. We apply a similar argument to express the 4 th and the 5 th sum on the right-hand side of (8). Finally as in [18], we substitute the new expressions for the 7th, 8th, 9th, and 11 th sum in (3) and use (2) to obtain

$$
\begin{aligned}
(\delta \theta(f)- & f \theta(L)+\theta(M) f)(x, y, z) \\
= & \widetilde{\sum}\left[\nu_{i}\left(f_{\alpha}(x), \nu_{j}\left(f_{\beta}(y), f_{\gamma}(z)\right)\right)-\nu_{i}\left(\nu_{j}\left(f_{\alpha}(x), f_{\beta}(y)\right), f_{\gamma}(z)\right)\right. \\
& \left.+\nu_{i}\left(\nu_{j}\left(f_{\alpha}(x), f_{\gamma}(z)\right), f_{\beta}(y)\right)\right],
\end{aligned}
$$

where

$$
\begin{aligned}
& \widetilde{\sum}=\sum_{\substack{i+\beta+\gamma+j=N+1 \\
\alpha=0, \beta, \gamma>0}}+\sum_{\substack{i+j+\alpha+\gamma=N+1 \\
\beta=0, \alpha, \gamma>0}} \\
& +\sum_{\substack{i+j+\alpha+\beta=N+1 \\
\gamma=0, \alpha, \beta>0}}+\sum_{\substack{i+j+\gamma=N+1 \\
\alpha=0=\beta, \gamma>0}}+\sum_{\substack{i+j+\beta=N+1 \\
\alpha=0=\gamma, \beta>0}} \\
& +\sum_{\substack{i+j+\alpha=N+1 \\
\beta=0=\gamma, \alpha>0}}+\sum_{\substack{i+j+\alpha+\beta+\gamma=N+1 \\
\alpha, \beta, \gamma>0}} .
\end{aligned}
$$

It follows from $(i i)$ that $(\delta \theta(f)-f \theta(L)+\theta(M) f)(x, y, z)=0$.

Theorem 5.5. Let $\left(\mu_{t}, \nu_{t} ; f_{t}\right)$ be a deformation of $f$ of order $N$. Then $\left(\mu_{t}, \nu_{t} ; f_{t}\right)$ extends to a deformation of order $N+1$ if and only if the cohomology class of $\theta\left(\mu_{t}, \nu_{t} ; f_{t}\right)$ vanishes.

Proof. Suppose that a deformation $\left(\mu_{t}, \nu_{t} ; f_{t}\right)$ of $f$ of order $N$ extends to a deformation of $f$ of order $N+1$. Then $(i)-($ iii $)$ hold for $n=N+1$. As a result, we get $\theta(L)=\delta \mu_{N+1}, \theta(M)=\delta \nu_{N+1}$, and $\theta(f)=f \mu_{N+1}-\nu_{N+1} f-\delta f_{N+1}$. In other 
words, the obstruction cochain $\theta\left(\mu_{t}, \nu_{t} ; f_{t}\right)=d\left(\mu_{N+1}, \nu_{N+1} ; f_{N+1}\right)$. So its cohomology class vanishes. Conversely, let $\theta\left(\mu_{t}, \nu_{t} ; f_{t}\right)$ be a coboundary. Suppose that

$$
\theta\left(\mu_{t}, \nu_{t} ; f_{t}\right)=d\left(\mu_{N+1}, \nu_{N+1} ; f_{N+1}\right)
$$

for some 2-cochain $\left(\mu_{N+1}, \nu_{N+1} ; f_{N+1}\right) \in C L^{2}(f ; f)$. Set

$$
\left(\tilde{\mu}_{t}, \tilde{\nu}_{t} ; \tilde{f}_{t}\right)=\left(\mu_{t}+\mu_{N+1} t^{N+1}, \nu_{t}+\nu_{N+1} t^{N+1} ; f_{t}+f_{N+1} t^{N+1}\right) .
$$

Then $\left(\tilde{\mu}_{t}, \tilde{\nu}_{t} ; \tilde{f}_{t}\right)$ satisfies $(i)-(i i i)$ for $0 \leqslant n \leqslant N+1$, so $\left(\tilde{\mu}_{t}, \tilde{\nu}_{t} ; \tilde{f}_{t}\right)$ is an extension of $\left(\mu_{t}, \nu_{t} ; f_{t}\right)$ of order $N+1$.

Corollary 5.6. If $H L^{3}(f ; f)=0$, then every 2 -cocycle in $C L^{2}(f ; f)$ is the infinitesimal of some deformation of $f$.

\section{Acknowledgements}

I wish to express my gratitude to Professor Goutam Mukherjee for his guidance. I would also like to thank the referee for his valuable comments and suggestions for improving the paper.

\section{References}

[1] D. Balavoine, Deformations of algebras over a quadratic operad. Operads: Proceedings of Renaissance Conferences, 207-234, Contemp. Math. 202, Amer. Math. Soc., Providence, RI, 1997.

[2] M. Gerstenhaber, The cohomology structure of an associative ring, Ann. of Math. 78, (1963), 267-288.

[3] M. Gerstenhaber, On the deformation of rings and algebras, Ann. of Math. 79 (1964), 59-103.

[4] M. Gerstenhaber, On the deformation of rings and algebras. II, Ann. of Math. 84 (1966), 1-19.

[5] M. Gerstenhaber On the deformation of rings and algebras. III, Ann. of Math. 88 (1968), 1-34.

[6] M. Gerstenhaber, On the deformation of rings and algebras. IV, Ann. of Math. 99 (1974), 257-276.

[7] M. Gerstenhaber and S.D. Schack, On the deformation of algebra morphisms and diagrams, Trans. Amer. Math. Soc. 279 (1983) No. 1, 1-50.

[8] M. Gerstenhaber and S.D. Schack, On the cohomology of an algebra morphism, J. Algebra 95 (1985), 245-262.

[9] M. Gerstenhaber and S.D. Schack, Sometimes $H^{1}$ is $H^{2}$ and discrete groups deform, in: Geometry of group representations, Contemp. Math. 74 (1988), Amer. Math. Soc., Providence, RI, 149-168.

[10] J.-L. Loday, Une version non commutative des algèbres de Lie: les algèbres de Leibniz, Enseign. Math. 39 (1993), No. 3-4, 269-293. 
[11] J.-L. Loday, Overview on Leibniz algebras, dialgebras and their homology. Cyclic cohomology and noncommutative geometry, 91-102, Fields Inst. Commun., 17, Amer. Math. Soc., Providence, RI, 1997.

[12] J.-L. Loday, Dialgebras, Dialgebras and related operads, 7-66, Lecture Notes in Math., 1763, Springer, Berlin, 2001.

[13] J.-L. Loday and T. Pirashvili, Universal enveloping algebras of Leibniz algebras and (co)homology, Math. Ann. 296 (1993), 139-158.

[14] A. Majumdar and G. Mukherjee, Deformation theory of dialgebras, $K$-Theory 27 (2002), 33-60.

[15] A. Nijenhuis and R.W. Richardson, Cohomology and deformations in graded Lie algebras, Bull. Amer. Math. Soc. 72 (1966), 1-29.

[16] A. Nijenhuis and R.W. Richardson, Deformations of homomorphisms of Lie algebras, Bull. Amer. Math. Soc. 73 (1967), 175-179.

[17] D. Yau, Deformation of dual Leibniz algebra morphisms, to appear in Comm. Algebra, arXiv:math.QA/0602046 v1.

[18] D. Yau, Deformation theory of dialgebra morphisms, to appear in Algebra Colloq., arXiv:math.RA/0604404 v1.

Ashis Mandal ashis_r@isical.ac.in

Stat-Math Unit

Indian Statistical Institute

203 B. T. Road

Kolkata-700108

India

This article is available at http://intlpress.com/HHA/v9/n1/a17 